\title{
Deep Donor Levels in Semiconductors with Vacancies in the Anion Sublattice
}

\author{
M.I. Daunov, R.R. Bashirov and G.M. Gajiev* \\ Laboratory of high pressure physics, Amirkhanov Institute of Physics Federal Research Center, \\ Russian Academy of Sciences, 367015 Makhachkala, Russia
}

(Received September 26, 2019; revised version December 4, 2019; in final form December 11, 2019)

The impurity electron spectrum for undoped bulk crystals of $n$-type arsenides GaAs, InAs, CdSnAs $2, \mathrm{CdGeAs}_{2}$, and $\mathrm{CdTe}, \mathrm{ZnO}$ has been studied by quantitative analysis of baric and temperature dependences of kinetic coefficients. The vacancies in the anion sublattice in these semiconductors have been found to be corresponding to deep donor centers for following reasons. Shallow intrinsic centers at hydrostatic pressure are dependent on their own band. In contrast, the energy of deep impurity centers at isotropic compression of crystal lattice is constant relative to absolute vacuum. The energy level locations relative to the conduction band edge and the pressure coefficients for energy spans between them and corresponding bottoms of conduction bands have been determined Energy levels of deep donor centers shift into the depth of conduction band with a decrease of the band gap.

DOI: 10.12693/APhysPolA.137.379

PACS/topics: hydrostatic pressure, pressure coefficient, Brillouin zone, deep donor

\section{Introduction}

As it is known $[1,2]$, the electronic conductivity in a binary III-V and ternary II-IV- $\mathrm{V}_{2}$ undoped semiconductors of InAs, GaAs, $\mathrm{CdSnAs}_{2}$, and $\mathrm{CdGeAs}_{2}$ is caused by a compound nonstoichiometry stipulated by vacancies in anionic sublattices, as arsenic is a highly volatile component. This conclusion has been confirmed by the study of nature of defects in arsenide crystals, where deep donor centers have been found [3]. Under hydrostatic pressure the shallow impurity centers shift together with their conduction band. In contrast, the energy of deep impurity centers are kept constant relative to absolute vacuum at isotropic compression of crystal lattice [4-7]. This invariance is due to the fact that wave functions of localized states should be built over whole Brillouin zone, and influence of the hydrostatic pressure on energy of states is defined by evolution of total energy spectrum, and not by first or second nearest bands only [8-10].

Note that it is difficult to determine the nature of impurity center from its phenomenological description based on energy of ionization, capture section, etc. at atmospheric pressure [4-7]. Therefore, the study of the energy spectrum evolution for charge carriers in semiconductors at hydrostatic pressure seems to be productive.

In this work, the results of the quantitative analysis based on the experimental data on electronic transport under hydrostatic pressure are presented for the purpose of deeper study of the impurity energy spectrum in the arsenides InAs, GaAs, $\mathrm{CdSnAs}_{2}$, and $\mathrm{CdGeAs}_{2}$. The comparison has been made with calculated data on $\mathrm{ZnO}$ and CdTe of II-VI groups.

*corresponding author; e-mail: hadzhygm@mail.ru
All samples presented in this work was prepared and measured out as follows. Undoped InAs and GaAs single crystals were grown by liquid encapsulated Czochralski method (LEC). CdSnAs 2 and $\mathrm{CdGeAs}_{2}$ crystals were prepared by a vertical Bridgman process. The growth charge was synthesized from a stoichiometric mixture of fine $\mathrm{CdAs}_{2}$ and Ge or Sn powders obtained by grinding high-purity (99.99999+\%) single crystals. In addition, the mixture contained an excess of arsenic, calculated from the free volume of the growth ampoule. The process was run under a vacuum of $10^{-2} \mathrm{~Pa}$ in graphitized ampoules with a capillary side arm, which contained a single-crystal required seed.

Undoped $\mathrm{ZnO}$ single crystals were grown by the hydrothermal method in concentrated alkali solutions on monohedral seeds at the crystallization temperature of $300-35^{\circ} \mathrm{C}$, under pressure of $30-50 \mathrm{MPa}$, with the direct temperature difference of $6-20^{\circ} \mathrm{C}$ between the growth and solution chambers of the autoclave. Undoped CdTe single crystals had been synthesized by growing technology for compounds II-VI from a melt by vertical zone melting under high pressure, designed to produce binary (ZnS, ZnSe, ZnTe, CdS, CdSe, CdTe) crystals.

The Hall coefficient $R_{\mathrm{H}}$ and resistivity $\rho$ depending on hydrostatic pressure at ambient temperature were measured for all objects. The hydrostatic pressure $P \leq 9 \mathrm{GPa}$ was produced in a high-pressure device of "flat anvils with holes and a toroid-shaped support". A teflon ampoule containing a sample, filled with pressure transmitting liquid, was placed in a hole of the lithographic stone gasket and compressed by two solid alloy anvils. The well-known mixture of 4:1 methanol and ethanol with a satisfactory level of hydrostatic character up to $10 \mathrm{GPa}$, was used as a medium, transmitting pressure. The pressure in the chamber was measured by a manganin sensor calibrated against $\mathrm{Bi}, \mathrm{Ta}$, and other standards. More details of the measurement technique can be found in the corresponding references. 


\section{Results and discussions}

The quantitative analysis of experimental results measured at room temperatures has been carried out for two reasons. Firstly, in this case one may neglect a broadening of deep levels [11]. Secondly, as it has been noted in $[12-14]$ the chaotic potential influence on band structure is enlarged with decrease of the temperature and free carrier concentration resulting in calculation mistakes for pressure coefficients. The known data on the dispersion law, effective mass of electrons at bottom of a conductivity band $m_{n}$, width of the band gap $\varepsilon_{\mathrm{g}}$, baric coefficient $\mathrm{d} \varepsilon_{\mathrm{g}} / \mathrm{d} P$ have been used in calculations.

Calculations have been done taking into account the statement about fixed level of deep impurity energy, measured from electron affinity level [4-7] when hydrostatic pressure is changed and with the use of next expressions $[15]$ :

$$
\begin{aligned}
& n_{d j}=\frac{N_{d}}{1+\beta \exp \left(\varepsilon_{d j}^{*}-\eta_{j}\right)} \\
& \ln \left(\frac{N_{d j}}{n_{d j}}-1\right)+\eta_{j}=\varepsilon_{d 0}^{*}-\left(\frac{\mathrm{d} \varepsilon_{\mathrm{g}}}{\mathrm{d} P}\right) P_{j}+\ln \beta \\
& N=n_{j}+n_{d j}=N_{d}+N_{s h}-N_{a}, \\
& \frac{n_{1}-n_{3}}{n_{1}-n 2}=\frac{n_{d 3}-n_{d 1}}{n_{d 2}-n_{d 1}} \\
& \beta \exp \varepsilon_{d}^{*}=(1-A)\left[A \exp \left(\left(P_{1}-P_{3}\right)\left(\frac{\mathrm{d} \varepsilon_{\mathrm{g}}}{\mathrm{d} P}\right)^{*}-\eta_{3}\right)\right. \\
& \left.\quad-\exp \left(\left(P_{1}-P_{2}\right)\left(\frac{\mathrm{d} \varepsilon_{\mathrm{g}}}{\mathrm{d} P}\right)^{*}-\eta_{2}\right)\right]^{-1},
\end{aligned}
$$

where

$$
A=\frac{n_{1}-n_{3}}{n_{1}-n 2} \frac{\exp \left(\left(P_{1}-P_{2}\right)\left(\frac{\mathrm{d} \varepsilon_{g}}{d P}\right)^{*}+\eta_{1}-\eta_{2}\right)-1}{\exp \left(\left(P_{1}-P_{3}\right)\left(\frac{\mathrm{d} \varepsilon_{g}}{\mathrm{~d} P}\right)^{*}+\eta_{1}-\eta_{3}\right)-1} .
$$

Indexes $0,1,2,3$ identify the ambient and operative pressure values of $P_{1}<P_{2}<P_{3}$, respectively, $\varepsilon_{d j}(j=1,2,3)$ is reduced energy between the conductivity band bottom and deep donor level energy, $\eta_{j}(j=1,2,3)$ is reduced Fermi's energy, and $\left(\mathrm{d} \varepsilon_{\mathrm{g}} / \mathrm{d} P\right)^{*}$ is baric coefficient for band gap at ambient pressure. $n_{1}, n_{2}, n_{3}$ and $n_{d 1}$, $n_{d 2}, n_{d 3}$ are concentrations of electrons in the conductivity band and electrons bound up with deep donors, $N_{d}$ and $N_{s h}$ are concentrations of the deep and shallow donors, respectively, $\beta$ is parameter of spin degeneration.

Unlike the wide band semiconductors, where the spinorbit splitting value is $\Delta \approx \varepsilon_{\mathrm{g}}$ and two-band approximation may be reasonable, for $n$-InAs, $n$-CdSnAs 2 and $n$ $\mathrm{CdGeAs}_{2}$ one needs issue the three band Kane model [15] as $\Delta$ and $\varepsilon_{\mathrm{g}}$ are close to the values

$$
m_{n}=\frac{\varepsilon_{\mathrm{g}}\left(\varepsilon_{\mathrm{g}}+\Delta\right)}{\varepsilon_{\mathrm{g}}+\frac{2}{3} \Delta} \frac{\hbar^{2}}{2 P_{M}^{2}}=\frac{3 \hbar^{2} \varepsilon_{\mathrm{geff}}}{4 P_{M}^{2}},
$$

where $P_{M}$ is the matrix element taking into account the interaction between conductivity and valence bands, $\hbar$ is reduced Planck constant.
Effective band gap and effective electron mass in the listed above semiconductors are (here and after $P$ is taken in GPa units):

$$
\begin{aligned}
& \text { - } \mathrm{CdSnAs}_{2}-\varepsilon_{\text {geff }}^{*}=(0.2+0.09 P) \mathrm{eV}, \\
& m_{n} / m_{0}=0.016+7.4 \times 10^{-3} P ; \\
& \text { - } \mathrm{InAs}-\varepsilon_{\text {geff }}^{*}=(0.322+0.077 P) \mathrm{eV}, \\
& m_{n} / m_{0}=0.022+5.24 \times 10^{-3} P \\
& \text { - } \mathrm{CdGeAs}_{2}-\varepsilon_{\text {geff }}^{*}=(0.405+0.065 P) \mathrm{eV}, \\
& \\
& m_{n} / m_{0}=0.02+3.24 \times 10^{-3} P .
\end{aligned}
$$

\subsection{Indium and gallium arsenides, $n$-InAs and $n-G a A s$}

Typical baric dependences for the Hall coefficient $R_{\mathrm{H}}(P)$, resistancy $\rho(P)$, and mobility $\mu_{\mathrm{H}}=\left|R_{\mathrm{H}}\right| / \rho$ for $n$-InAs single crystal with concentration of excess donors of $\approx 10^{16} \mathrm{~s} \mathrm{~m}^{-3}$ at $300 \mathrm{~K}$ are presented in Fig. 1 [16]. $R_{\mathrm{H}}$ practically does not depend on pressure in the range till to (2-3) GPa. Then $\left|R_{\mathrm{H}}\right|$ and $\rho$ increase with pressure with the exponential law up to $P \approx 6.5 \mathrm{GPa}$ (the beginning of polymorphic transition). Such character of the $R_{\mathrm{H}}(P)$ dependence in the 2.5-6 GPa range is caused by presence of the deep resonant donor impurity centers: vacancies of arsenic with concentration of $N_{d}[3,17]$. We notice that in InAs, extrema of $L$-valley and $X$-valley are located above the $\Gamma$-valley extremum, by $\approx 1 \mathrm{eV}$ and $\approx 1.5 \mathrm{eV}$, accordingly [18].

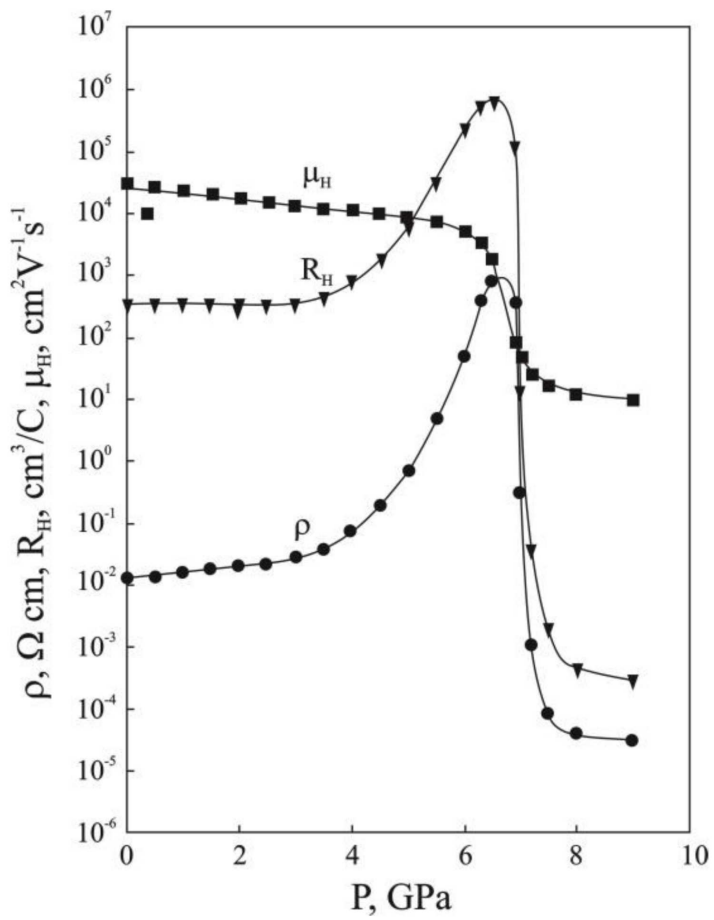

Fig. 1. Baric dependences of resistivity (-๑-), the Hall coefficient $(-\mathbf{\nabla}-)$ and the Hall mobility (-ם-) of electrons in the $n$-InAs single crystal sample at $T=300 \mathrm{~K}$ with concentration of electrons at ambient pressure $n=1.84 \times 10^{16} \mathrm{~s} \mathrm{~m}^{-3}[16]$. 


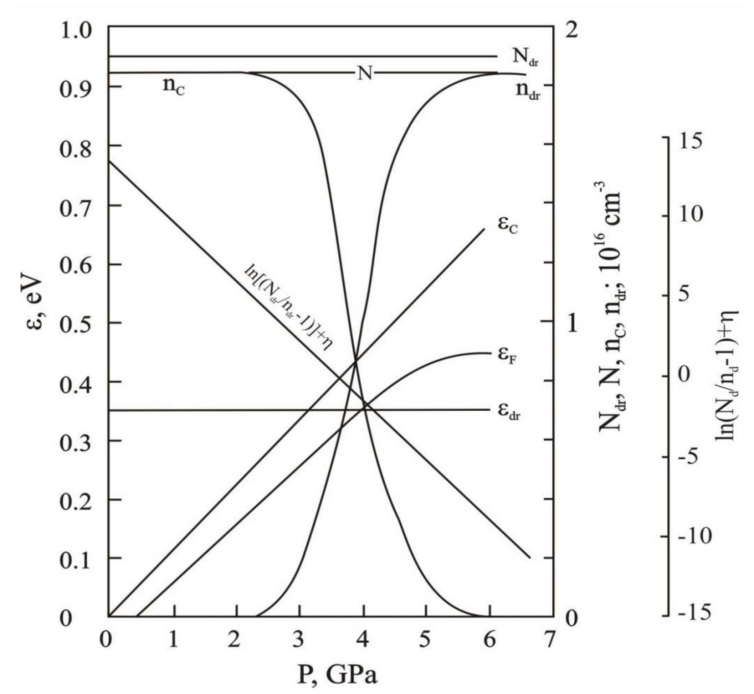

Fig. 2. Baric dependences for energy $\varepsilon_{c}$ of the conductivity band bottom, the level of deep resonant donor $\varepsilon_{d r}$, Fermi's energy $\varepsilon_{\mathrm{F}}$ relatively to $\varepsilon_{C 0}(P=0)$, concentration of electrons in conductivity band $n$ and electrons located at the deep donor centers $n_{d r}$, and function $\ln \left(\left(N_{d r} / n_{d r}-1\right)\right)+\eta$ in $n$-InAs at $T=300 \mathrm{~K}$ with concentration of electrons at ambient pressure $n=1.84 \times 10^{16} \mathrm{~s} \mathrm{~m}^{-3} . \quad N=n+n_{d r}, N_{d r}$ is concentration of the deep donor centers.

Our results concerning $R_{H}(P)$ (Fig. 1) for the sample with $n_{0}=1.84 \times 10^{16} \mathrm{~s} \mathrm{~m}^{-3}$ are presented in Fig. 2 . According to relations (1)-(4):

$$
\begin{aligned}
& \ln \left(\frac{N_{d r}}{n_{d r}}-1\right)+\eta= \\
& 13.43-4.26 P=\varepsilon_{d r 0}^{*}+\left(\frac{\mathrm{d} \varepsilon_{\mathrm{g}}}{\mathrm{d} P}\right)^{*} P+\ln \beta .
\end{aligned}
$$

Thus, we have obtained: $N_{d r}=1.9 \times 10^{16} \mathrm{~s} \mathrm{~m}^{-3}, N_{s h}-$ $N_{a}=-0.06 \times 10^{16} \mathrm{~s} \mathrm{~m}^{-3}$. For $\beta=1$ we obtain [6]:

$$
\varepsilon_{d r}=(0.35-0.11 P) \mathrm{eV} \quad(P-\text { in } \mathrm{GPa}) .
$$

Free electrons are completely localized at the donor centers and Fermi's energy is close to $\varepsilon_{d r}$ if $P>4.5 \mathrm{GPa}$. Position of the Fermi level at $P>5 \mathrm{GPa}$ has been stable relatively to resonant donor level (Fig. 2), and $\varepsilon_{\mathrm{F}}>\varepsilon_{d r}$ : $\varepsilon_{\mathrm{F}}-\varepsilon_{d r} \approx 0.09 \mathrm{eV}$.

In the volume crystals of $n$-GaAs with concentration of excess donors $N_{d}=\left(1.8 \times 10^{16} \div 5.5 \times 10^{17}\right) \mathrm{s} \mathrm{m}^{-3}$ the presence of deep donor level with $\varepsilon_{d}=(-0.15+$ $\left.1.1 \times 10^{-7} N_{d}^{1 / 3}\right) \mathrm{eV}$ relative to the $\Gamma$-valley was revealed from $\rho(T)$ and $R_{H}(T)$ temperature dependences at atmospheric pressure [19].

\section{2. $n-C d S n A s_{2}$ and $n-C d G e A s_{2}$}

These materials, being cognate by their properties, belong to the most studied semiconductor of II-IV-V group [20, 21]. Their band structures are similar to each other. However, electron mobility in $n$-CdGeAs 2 is much less in comparison to that in $n$-CdSnAss $\mathrm{C}_{2}$. Also

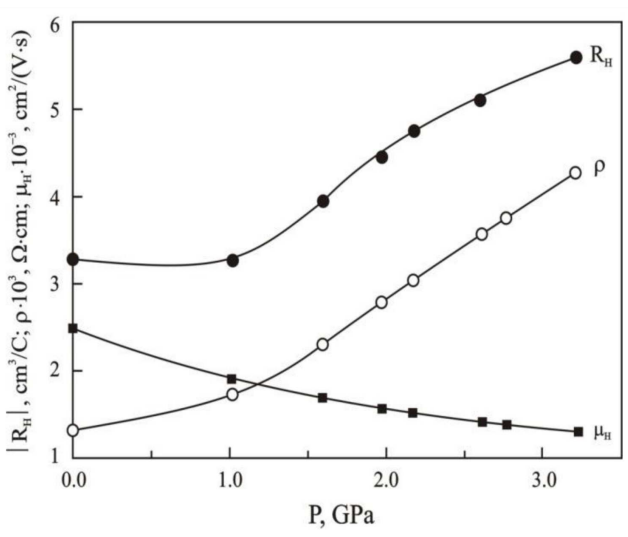

Fig. 3. Dependences of resistivity(-O-), Hall coefficient (--) and the Hall mobility (- - -) of electrons on pressure in the single crystal $n$-CdSnAs 2 at $T=300 \mathrm{~K}$ with concentration of electrons at ambient pressure $n=1.9 \times 10^{18} \mathrm{~s} \mathrm{~m}^{-3}$.

some vagueness exists in experimental data for baric dependence of resistivity in $n$-CdGeAs $s_{2}$. The conclusion about existence of additional subbands in the $n$ $\mathrm{CdGeAs}_{2}$ and $\mathrm{CdSnAs}_{2}$ conductivity band has been drawn (see [20,21] and references therein) on the basis of the optical absorption, Faraday effect, photoconductivity, and kinetic phenomena data. Nevertheless, theoretical calculations $[22,23]$ have not confirmed any presence of additional minima in the conductivity band close to the main minimum, and observed features in experimental data are caused apparently by an impurity spectrum.

$R_{\mathrm{H}}(P)$ and $\rho(P)$ dependences for $n$-InAs (Fig. 1) and $n$-CdSnAs 2 (Fig. 3) [24] are similar. It is due to the presence of a deep donor center, energy level of which falls in the continuum of the conductivity band in both materials. The pressure distinction, when these dependences are sharply growing, is caused by the fact that initial concentration of electrons in the investigated samples differ by two orders of magnitude. For $n$-InAs $n=1.84 \times 10^{16} \mathrm{~s} \mathrm{~m}^{-3}, n-\mathrm{CdSnAs}_{2} n=1.9 \times 10^{18} \mathrm{~s} \mathrm{~m}^{-3}$ and their Fermi's energy values at atmospheric pressure are $\varepsilon_{\mathrm{F}}=-0.04 \mathrm{eV}$ and $\varepsilon_{\mathrm{F}}=0.19 \mathrm{eV}$, accordingly. Also, an increase in resistivity with the pressure in $n$ InAs is much more noticeable than that in $n$-CdSnAs 2 (Figs. 1 and 3).

In the investigated $n$-CdSnAs $\mathrm{C}_{2}$ crystals, concentration of electrons practically does not depend on pressure until $P=1 \mathrm{GPa}$ (a growth within the limits $(1-2) \%$ is caused by volume-concentration effect [25]), and growth of $\rho(P)$ is connected with a decrease of the electron mobility [15].

Taking into account experimental dependence on the pressure for kinetic coefficients in the range of 1-3.4 GPa (Fig. 3), initial concentration of electrons $n=1.9 \times$ $10^{18} \mathrm{~s} \mathrm{~m}^{-3}$, the $\chi(P)$ dependence [21], calculated $m_{n}(P)$ and $m_{n}(\eta)$ dependences, the $n(P)$ and $\eta(P)$ dependences have been defined with the use of known values of band parameters and effective width of the band gap (4) in two-band approximation of Kane model [15]. 


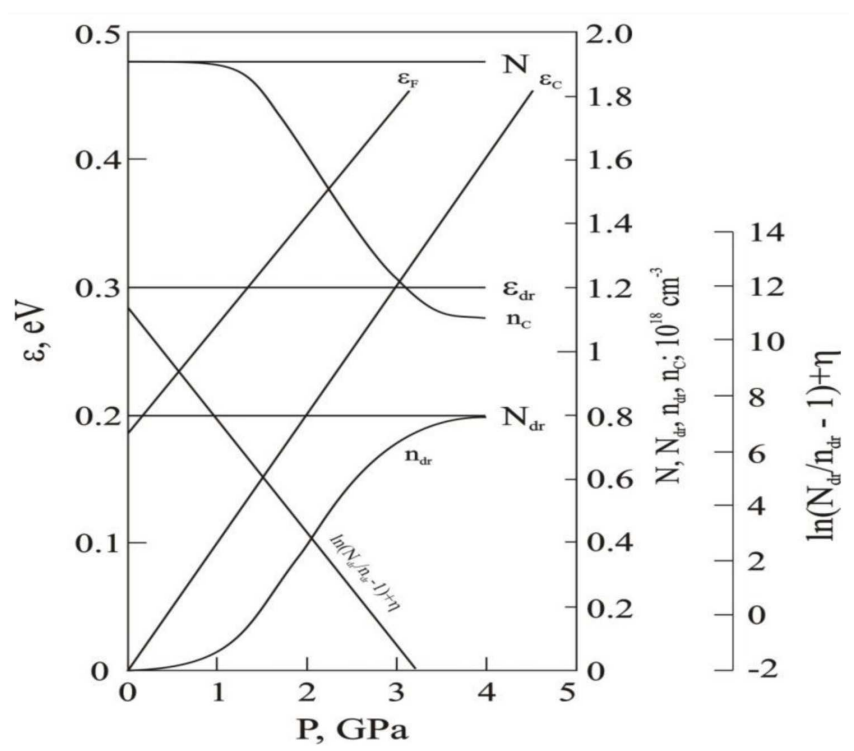

Fig. 4. Pressure dependences for the conductivity band bottom $\varepsilon_{c}$, the Fermi energy $\varepsilon_{\mathrm{F}}$, energy of deep resonant donor $\varepsilon_{d r}$ relative to $\varepsilon_{C 0}(P=0)$, and concentrations of electrons in conductivity band $n$, and electrons located at deep donor centers $n_{d r}$, and function $\ln \left(\left(N_{d r} / n_{d r}-1\right)\right)+\eta$ for $n$-CdSnAs 2 with concentration of electrons at atmospheric pressure $n=1.9 \times 10^{18} \mathrm{~s} \mathrm{~m}^{-3}$ and $T=300 \mathrm{~K} . N_{d r}$ and $N_{s h}$ are concentrations of deep and shallow donor, $N_{a}$ is concentration of compensating acceptors $\left(N=n+n_{d r}=N_{d r}+N_{s h}-N_{a}\right)$.

By means of $n(P)$ and $\eta(P)$ dependences, according to (1) $-(3)$ one obtains

$\ln \left(\frac{N_{d r}}{n_{d r}}-1\right)+\eta=11.8-4.14 P=\varepsilon_{d r 0}^{*}+\left(\frac{\mathrm{d} \varepsilon_{\mathrm{g}}}{\mathrm{d} P}\right)^{*} P$.

Thus, it was found: $N_{d r}=8 \times 10^{17} \mathrm{~s} \mathrm{~m}^{-3}, N_{s h}-N_{a}=$ $1.1 \times 10^{18} \mathrm{~s} \mathrm{~m}^{-3}$. For $\beta=1$ :

$$
\varepsilon_{d r}=(0.3-0.11 P) \mathrm{eV} \quad(P-\text { in GPa }) .
$$

In Fig. 4, the results of the quantitative analysis are presented. Unlike the $n$-InAs sample with $N=1.84 \times$ $10^{16} \mathrm{~s} \mathrm{~m}^{-3}$ and $N_{s h}-N_{a}=-0.06 \times 10^{16} \mathrm{~s} \mathrm{~m}^{-3}$ (Figs. 1 and 2), in the $n-\mathrm{CdSnAs}_{2}$ sample with $N=$ $1.9 \times 10^{18} \mathrm{~s} \mathrm{~m}^{-3}$ we have $\left(N_{s h}-N_{a}\right)=1.1 \times 10^{18} \mathrm{~s} \mathrm{~m}^{-3}$. In $n$-CdSnAs2, $n \rightarrow N_{s h}-N_{a}, n_{d r} \rightarrow N_{d r}$, and in $n$-InAs, $n \rightarrow 0, n_{d r} \rightarrow N$ if $P \rightarrow \infty$. These calculated features explain, why $\rho$ increases prior to the beginning of a phase transition more than by four orders of magnitude in $n$ InAs, and $\rho$ in $n$-CdSnAs 2 increases but by $\approx 5$ times (Figs. 1 and 3 ).

Experimental data for $\rho(P)$ in $n$-CdGeAs 2 are inconsistent (Fig. 5) [26, 27] up to $P=1 \mathrm{GPa}$ at $300 \mathrm{~K}$. In crystals doped by $\mathrm{Cd}$ and Te with concentration of $n=\left(10^{17} \div 10^{18}\right) \mathrm{s} \mathrm{m}^{-3}$ we have $\rho(P) / \rho(0)=$ $1.22 \div 1.24 \quad(P=1 \mathrm{GPa})[26]$. An increase of $\rho$ at $0 \leq P \leq 1 \mathrm{GPa}$, exactly like that in $n$-CdSnAs 2 , is caused by a decrease of an electron mobility and

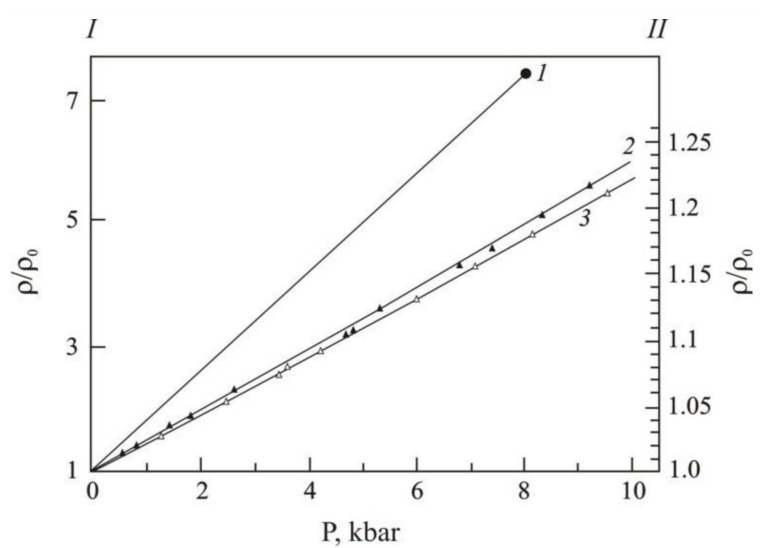

Fig. 5. Dependences of resistivity on pressure at 300 $\mathrm{K}[27]$ in $n$-CdGeAs 2 undoped (1 --) (axis I) [26] and doped by tellurium (2 - $\left.\boldsymbol{\Delta}^{-}\right)$and indium $\left(3-\triangle^{-}\right)$(axis II) samples.

matches with the pressure dependences of the band gap $\partial \varepsilon_{\mathrm{g}} / \partial P=0.093 \mathrm{eV} \mathrm{GPa}^{-1}[28]$ and static dielectric permeability (Figs. 1 and 5). One can notice that a decrease of $\chi$ with the pressure growth has an essential influence on $\rho(P) \approx \mu(P)^{-1}$ and should be taken into account for correct interpretation of experimental data.

In undoped $n$-CdGeAs 2 , we have $\rho / \rho_{0}=7$ at $P=0.8 \mathrm{GPa}$ [27] (Fig. 5). Such a strong increase of $\rho(P)$ in this material is well correlated with the presence of the deep donor level (vacancy of arsenic) near the conductivity band edge: $\varepsilon_{d}=(-0.05-0.093 P) \mathrm{eV}$. This result is in agreement with [3] where $\mathrm{CdGeAs}_{2}$ exposed to the irradiation has been investigated, and the presence of donors with $\varepsilon_{d}=-0.05 \mathrm{eV}$ has been found. For concentration of electrons at ambient pressure $n=10^{17} \mathrm{~s} \mathrm{~m}^{-3}$ we have

$$
\begin{aligned}
& N_{d}=1.65 \times 10^{18} \mathrm{~s} \mathrm{~m}^{-3}, \\
& N_{s h}-N_{a}=-3 \times 10^{16} \mathrm{~s} \mathrm{~m}^{-3}, \\
& \frac{\rho(P)}{\rho_{0}}=1+7.4 \times 10^{-14} .
\end{aligned}
$$

The results of the quantitative analysis are presented in Fig. 6.

\subsection{Comparison with deep donors in undoped $n-Z n O$ and $n-C d T e$}

It seems interesting to verify how the data on two other semiconductors falling out of a series of arsenides fit into the proposed concept, namely: semiconducting $\mathrm{ZnO}$ and CdTe, belonging to compounds of II-VI groups. Zinc oxide as a wide-band-gap semiconductor has a number of applications, for instance in varistors, phosphors, and sensors. It is known that nominally undoped $\mathrm{ZnO}$ has $n$ type and the source of this conductivity is due to intrinsic defects such as oxygen vacancies ( $\mathrm{V}_{\mathrm{O}}$ in anion sublattice) acting as deep donors [29]. It has been found that the oxygen vacancies in $\mathrm{ZnO}$ are actually +2 charged and 


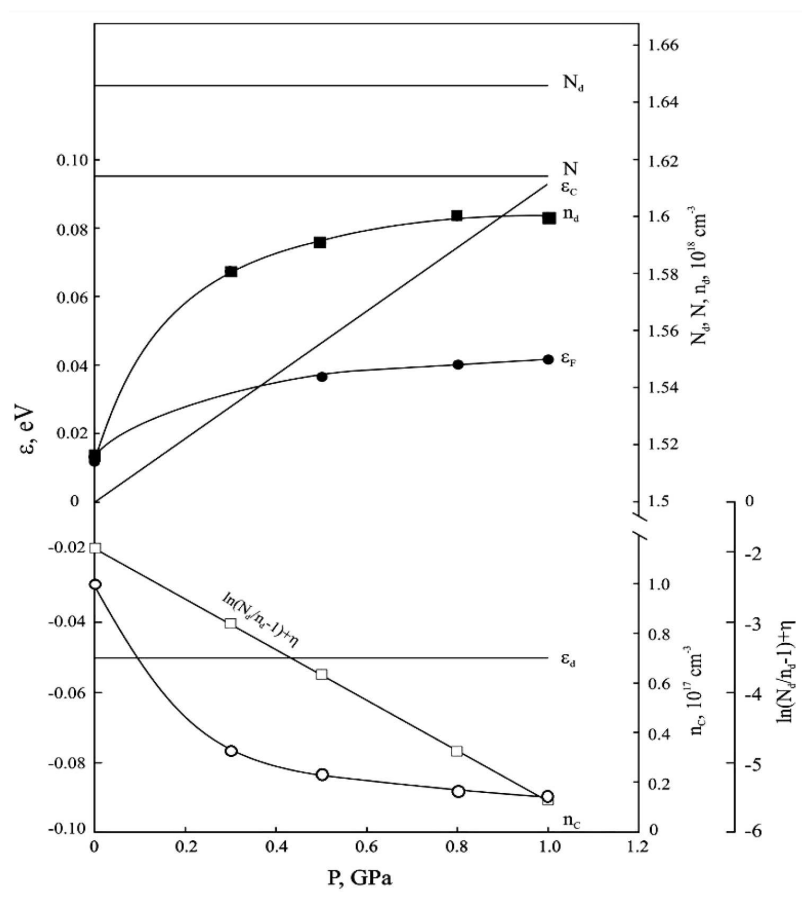

Fig. 6. Pressure dependences for the conductivity band bottom $\varepsilon_{C}$ (sloping solid line), the Fermi energy $\varepsilon_{\mathrm{F}}\left(-\bullet_{-}\right)$, energy level of the deep resonant donor centers $\varepsilon_{d}$, relative to $\varepsilon_{C 0}(P=0)$ (horizontal solid line), concentration of electrons in the conductivity band $n_{C}\left(-\mathrm{O}_{-}\right)$, electrons on the deep donor centers $n_{d}$ (-口-), and function $\ln \left(N_{d} / n_{d}-1\right)+\eta$ for $n$-CdGeAs$s_{2}$ with concentration of electrons at atmospheric pressure $n=10^{17} \mathrm{~s} \mathrm{~m}^{-3}$ at $T=300 \mathrm{~K}(-\square-) . N_{d r}$ and $N_{s h}$ are concentrations of deep and shallow donors, $N_{a}$ is concentration of compensating acceptors, $\left(N=n+n_{d}=\right.$ $\left.N_{d}+N_{s h}-N_{a}\right)$.

thus responsible for the unintentional $n$-type conductivity, negative $U$ behavior as well as the nonstoichiometry of $\mathrm{ZnO}$ [30]. Another semiconductor — undoped CdTe - synthesized under accidentally nonstoichiometric Cdrich conditions (some Te deficiency) can contain not only anionic vacancies, but also cationic interstitials [31].

The $n$-type deep centers, for which the energy levels are located under the conductivity band bottom, have been calculated from the experimental baric and temperature dependences of electron transport in the CdTe [32] and $n$ - $\mathrm{ZnO}$ [33] undoped bulk single crystals. It was found that $\varepsilon_{d}=0.112 \mathrm{eV}$ for $n-\mathrm{CdTe}$, and $\varepsilon_{d}=0.37 \mathrm{eV}$ for $n$ $\mathrm{ZnO}$. Four-level model for $n$-CdTe and three-level model for $n$ - $\mathrm{ZnO}$ were used.

\section{Conclusion}

In studied $n$-type semiconducting arsenides - GaAs, InAs, CdSnAs 2 , and $\mathrm{CdGeAs}_{2}$ - the As vacancies play a role of deep donor levels. Positions of these levels against the $\Gamma$-valley edge of conductivity band and pressure coefficients for these levels have been obtained.

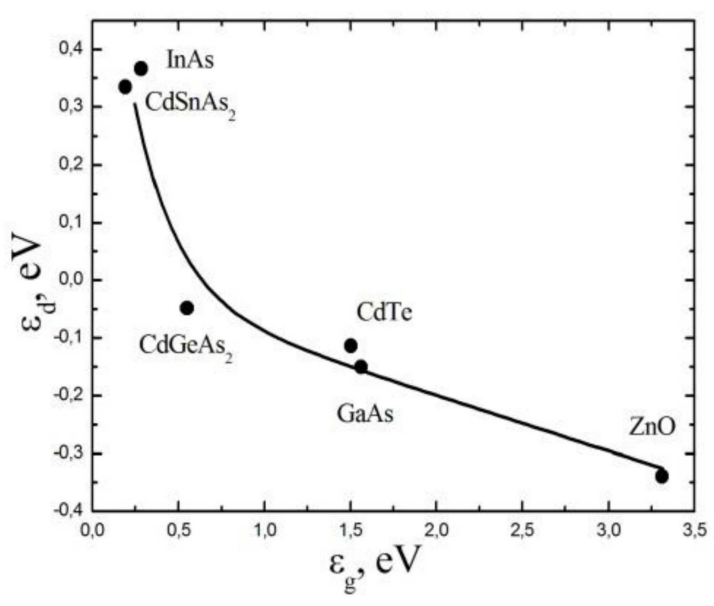

Fig. 7. The dependence of deep donor energy level relatively to $\Gamma$-valley bottom of conductivity band on the band gap width in semiconductors.

Figure 7 demonstrates the $\varepsilon_{d}=f\left(\varepsilon_{\mathrm{g}}\right)$ dependence of the deep donor center energies for arsenic vacancies relative to the conduction band bottom on the band gap widths for InAs, $\mathrm{CdSnAs}_{2}, \mathrm{CdGeAs}_{2}, \mathrm{GaAs}$ and CdTe [32] and $\mathrm{ZnO}$ [33]. In brief, the figure is the main point of the paper.

Thus, a shift of the deep donor impurity center down into the depth of conduction band with a decrease in the width of the band gap has been observed. Occurrence of deep donor impurity center is stipulated by vacancies in the anion sublattice of semiconductors mentioned above.

\section{Acknowledgments}

This work was performed as part of the state tasks of the Ministry of Science and Higher Education of Russia Nos. AAAA-A17-117021310361-0; AAAA-A17117021310366-5.

\section{References}

[1] O. Madelung, Semiconductors, Intrinsic Properties of Group IV Elements and III-V, II-VI, and I-VII Compounds, Springer, Berlin 1987.

[2] M. Kamińska, M. Skowroński, W. Kuszko, J. Lagowski, J. Parsey, H.C. Gatos, Czech. J. Phys. B 34, 409 (1984).

[3] V.N. Brudnyi, T.V. Vedernikova, Semiconductors 42, 34 (2008).

[4] M.I. Daunov, U.Z. Zalibekov, I.K. Kamilov, A.Yu. Mollaev, Semiconductors 48, 996 (2014).

[5] M.I. Daunov, I.K. Kamilov, A.B. Magomedov, S.F. Gabibov, Phys. Status Solidi B 235, 297 (2003).

[6] M.I. Daunov, I.K. Kamilov, S.F. Gabibov, Phys. Solid State 46, 1825 (2004).

[7] I.K. Kamilov, S.F. Gabibov, M.I. Daunov, A.Yu. Mollaev, Semiconductors 45, 1543 (2011). 
[8] W. Paul, in: Proc. 9th Int. Conf. on Semiconductors 1968 , p. 51.

[9] V.A. Telejkin, K.B. Tolpigo, Semiconductors 16, 1337 (1982).

[10] In-Hwan Chor, Y.Yu. Peter, Phys. Status Solidi B 211, 143 (1999).

[11] D. Menichellia, E. Borchi, J. Appl. Phys. 93, 390 (2005); Jun Xia, A. Mandelisa, J. Appl. Phys. 105, 103712 (2009).

[12] M.I. Daunov, I.K. Kamilov, A.B. Magomedov, A.Sh. Kirokosyan, Semiconductors 33, 31 (1999).

[13] I.K. Kamilov, M.I. Daunov, V.A. Elizarov, A.B. Magomedov, JETP 77, 92 (1993).

[14] M.I. Daunov, I.K. Kamilov, S.F. Gabibov, Dokl. Phys. 53, 115 (2008).

[15] B.M. Askerov, in: Electron Transport Phenomena in Semiconductors, World Sci., River Edge (NJ) 1994.

[16] I.K. Kamilov, M.I. Daunov, R.R. Bashirov, A.Yu. Mollaev, R.K. Arslanov, S.F. Gabibov, T.R. Arslanov, Phys. Status Solidi B 246, 586 (2009).

[17] S. Das Sarma, A. Madhukar, Solid State Commun. 38, 183 (1981).

[18] I. Vurgaftman, J.R. Meyer, L.R. Ram-Mohan, J. Appl. Phys. 89, 5815 (2001).

[19] G.D. Pitt, J. Lees, Phys. Rev. B 2, 4144 (1970).

[20] Semiconductors $A^{2} B^{4} C_{2}^{5}$, Eds. N.A. Goryunova, Yu.A. Valov, Sov. Radio, Moscow 1974 (in Russian).

[21] M.I. Daunov, A.B. Magomedov, A.E. Ramazanova, Russ. Phys. J. 29, 661 (1986).
[22] V.V. Aksenov, V.M. Petrov, Yu.I. Polygalov, V.A. Chaldyshev, Russ. Phys. J. 15, 1163 (1972).

[23] A.S. Poplavnoi, Yu.I. Polygalov, Russ. Phys. J. 24, 1139 (1981).

[24] A.Yu. Mollaev, I.K. Kamilov, M.I. Daunov, R.K. Arslanov, A.B. Magomedov, L.A. Saypulaeva, S.F. Gabibov, High Press. Res. 26, 445 (2006).

[25] M.I. Daunov, V.I. Danilov, A.B. Magomedov, Russ. Phys. J. 40, 457 (1997).

[26] L. Konczewicz, S. Porowski, I.K. Polushina, High Temp. High Press. 7, 716 (1975).

[27] M.K.R. Vyas, G.D. Pitt, J. Phys. C Solid State Phys. 7, L423 (1974).

[28] R. Bendorius, V.D. Prochukhan, A. Sileika, Phys. Status Solidi B 53, 745 (1972).

[29] V. Avrutin, D.J. Silversmith, H. Morkoç, Proc. IEEE 98, 1269 (2010).

[30] L. Liu, Z. Mei, A. Tang, A. Azarov, A. Kuznetsov, Q.-K. Xue, X. Du, Phys. Rev. B 93, 235305 (2016).

[31] A. Nagaoka, K. Nishioka, K. Yoshino, D. Kuciauskas, M.A. Scarpulla, Appl. Phys. Express 12, 081002 (2019).

[32] M.I. Daunov, A.S. Kovalev, A.Yu. Mollaev, A.B. Magomedov, Semiconductors 45, 43 (2011).

[33] M.I. Daunov, R.K. Arslanov, M.M. Gadjialiev, E.V. Kortunova, P.P. Khokhlachev, P.P. Shvansky, Semiconductors 40, 1255 (2006). 